\title{
Telomere length in different histologic types of ovarian carcinoma with emphasis on clear cell carcinoma
}

Elisabetta Kuhn ${ }^{1}$, Alan K Meeker ${ }^{1,2}$, Kala Visvanathan ${ }^{3,4}$, Amy L Gross $^{4}$, Tian-Li Wang ${ }^{2,5}$, Robert J Kurman ${ }^{1,2,5}$ and Ie-Ming Shih ${ }^{1,2,5}$

${ }^{1}$ Department of Pathology, Johns Hopkins School of Medicine, Baltimore, MA, USA; ${ }^{2}$ Department of

Oncology, Johns Hopkins School of Medicine, Baltimore, MA, USA; ${ }^{3}$ Department of Medical Oncology, Johns Hopkins School of Medicine, Baltimore, MA, USA; ${ }^{4}$ Department of Epidemiology, Johns Hopkins School of Public Health, Baltimore, MA, USA and ${ }^{5}$ Gynecology and Obstetrics, Johns Hopkins Medical Institutions, Baltimore, MA, USA

Ovarian carcinoma is composed of a heterogeneous group of tumors with distinct clinico-pathological and molecular features. Alteration of telomerase activity has been reported in ovarian tumors but the pattern of telomere length in their specific histological subtypes has not been reported. In this study, we performed quantitative telomere fluorescence in situ hybridization on a total of 219 ovarian carcinomas including $106 \mathrm{high}-$ grade serous carcinomas, 26 low-grade serous carcinomas, 56 clear cell carcinomas and 31 low-grade endometrioid carcinomas. The mean relative telomere length of carcinoma to stromal cells was calculated as a telomere index. This index was significantly higher in clear cell carcinoma compared with the other histologic types $(P=0.007)$. Overall there was no association between the telomere index and mortality, but when stratified by histologic types, the hazard ratio for death among women with clear cell carcinoma with a telomere index $>1$ was significantly increased at $4.93(95 \% \mathrm{Cl} 1.64-14.86, P=0.005)$ when compared with those with a telomere index $\leq 1$. In conclusion, our results provide new evidence that telomere length significantly differs by histologic type in ovarian carcinoma. Specifically, clear cell carcinomas have longer mean relative telomere lengths compared with the other histologic types and longer telomeres in clear cell carcinoma are associated with increased mortality suggesting that aberrations in telomere length may have an important role in the development and progression of this neoplasm.

Modern Pathology (2011) 24, 1139-1145; doi:10.1038/modpathol.2011.67; published online 15 April 2011

Keywords: clear cell carcinoma; FISH; telomere; ovarian cancer

Ovarian carcinoma, the most lethal gynecologic malignancy, is composed of several different histologic types. ${ }^{1-3}$ A new dualistic model of carcinogenesis has been proposed, which provides a conceptual framework for studying this complex group of tumors based on clinicopathological and molecular genetic features. ${ }^{4}$ Tumors are grouped into two broad categories designated type I and type II. Type I tumors include low-grade serous,

Correspondence: I-M Shih, MD, PhD, Department of Pathology, Johns Hopkins Medical Institutions, CRB-II, Rm:305, 1550 Orleans Street, Baltimore, MA 21231, USA.

E-mail: ishih@jhmi.edu

Received 13 December 2010; revised 20 January 2011; accepted 20 January 2011; published online 15 April 2011 low-grade endometrioid, clear cell and mucinous carcinomas that develop in a stepwise manner from well-established precursor lesions, such as endometriosis and borderline tumors. ${ }^{5}$ Type II tumors are composed of high-grade serous carcinoma, carcinosarcoma and undifferentiated carcinoma and appear to develop from intraepithelial carcinomas in the fallopian tube. ${ }^{6}$ Type I tumors are characterized by indolent behavior and harbor KRAS, BRAF, PIK3CA, CCNB1, ARID1A and PTEN mutations. ${ }^{2}$ In contrast, type II tumors are highly aggressive and unlike the type I tumors have a very high frequency of TP53 mutations. ${ }^{7,8}$ They rarely harbor the mutations that occur in the type I tumors and also differ molecularly from the type I tumors by having a high level of DNA copy number alterations. ${ }^{9}$ 
Telomeres are specialized chromosomal structures that are essential for maintaining the integrity of chromosomal ends. In normal cells, excessive telomere attrition triggers a DNA damage response that leads to cell cycle arrest. Cancer cells must maintain telomere integrity, either through telomerase-dependent or independent mechanisms, to continuously proliferate. ${ }^{10}$ Although maintaining a certain telomere length is essential for tumor cell survival and progression, cancer cells also exploit telomere shortening, as exaggerated telomere shortening leads to telomere dysfunction and chromosomal instability by promoting abnormal fusion and rearrangement of chromosomes. ${ }^{11}$ In fact, telomere shortening is an early event during tumor progression as it has been demonstrated in preinvasive lesions of several epithelial cancers, including prostate, breast and pancreatic carcinomas. ${ }^{12}$ Similarly, we have recently demonstrated telomere shortening in the majority of serous tubal intraepithelial carcinomas, suggesting that it is one of the earliest molecular changes associated with the development of pelvic high-grade serous carcinoma. ${ }^{13}$

Given the important role of telomere biology in the pathogenesis of cancer development, ${ }^{14}$ we hypothesized that telomere length might differ in type I and type II ovarian carcinomas considering their distinctly different molecular genetic features. Although telomerase gene expression and activity have been reported in ovarian cancer, ${ }^{15-18}$ only a few previous studies have examined global telomere lengths by Southern blot in a small numbers of ovarian cancers. ${ }^{19-22}$ The current study is notable in that it is the first to use direct in situ telomere assessment to comprehensively compare telomere length profiles among the major types of ovarian carcinoma and to correlate telomere length with the clinicopathological features of each type.

\section{Materials and methods}

\section{Study Population}

A total of 219 ovarian carcinomas from 212 patients were included in this study. There were 106 highgrade serous carcinomas, 26 low-grade serous carcinomas, 56 clear cell carcinomas and 31 lowgrade endometrioid carcinomas of the ovary. There were seven patients whose primary and recurrent high-grade serous carcinomas were available for analysis. This study was approved by the Institutional Review Board at the Johns Hopkins Medical Institutions. All tumor paraffin blocks were retrieved from the archival files of the Johns Hopkins Hospital except low-grade endometrioid and clear cell carcinomas, which were obtained from the National Taiwan University Hospital. The carcinomas were arranged in tissue microarrays to minimize the potential technical inconsistency related to in situ hybridization. Four micrometer thick paraffin sections were prepared for telomere fluorescence in situ hybridization. The clinical information including age, ethnic background, clinical stage, primary vs recurrent tumor were obtained from medical records.

\section{Telomere Fluorescence in situ Hybridization}

Telomere length in different types of ovarian carcinoma were assessed by telomere fluorescence in situ hybridization (FISH) using a method previously described. ${ }^{23}$ Briefly, de-paraffinized slides were hydrated through a graded series of ethanol followed by deionized water plus $0.1 \%$ Tween- 20 . Slides were then placed in citrate buffer, steamed for 14 min (Black and Decker Handy Steamer Plus; Black and Decker, Towson, MD, USA) and then placed in PBS with Tween (PBST) for $5 \mathrm{~min}$. Twenty-five $\mu \mathrm{l}$ of a Cy3-labeled telomere-specific peptide nucleic acid (PNA) $(0.3-\mu \mathrm{g} / \mathrm{ml}$ PNA in $70 \%$ formamide, $10 \mathrm{mmol} / \mathrm{l}$ Tris, $\mathrm{pH} 7.5, \quad 0.5 \% \mathrm{~B} / \mathrm{M}$ Blocking reagent) was applied to the samples and incubated at $83^{\circ} \mathrm{C}$ for $4 \mathrm{~min}$. Slides were then moved to a dark, closed chamber for hybridization at room temperature for $2 \mathrm{~h}$. After two washes in a solution of $70 \%$ formamide, $10 \mathrm{mmol} / \mathrm{l}$ Tris, $\mathrm{pH} 7.5,0.1 \%$ albumin, slides were counterstained with 4'-6diamidino-2-phenylindole (DAPI). The PNA probe complementary to the mammalian telomere repeat sequence (5'-CCCTAACCCTAACCCTAA-3') was obtained from Applied Biosystems (Framingham, $\mathrm{MA}$ ), with an $N$-terminal covalently linked to Cy3 fluorescent dye. As a positive control for hybridization, a FITC-labeled PNA probe (5'-ATTCGTTGG AAACGGGA-3') specific for human centromeric DNA repeats (CENP-B binding sequence) was also included in the hybridization solution. ${ }^{24}$

\section{Microscopy and Image Analysis}

To measure the relative changes in telomere length, slides were imaged with a Nikon 50i epifluorescence microscope equipped with fluorescence excitation/emission filters for different (fluorophores) (Omega Optical). Grayscale images were captured using Nikon NIS-Elements software and an attached Photometrics Cool snap EZ digital camera. For presentation purposes, images were pseudo-colored and merged. Quantitative FISH was performed on the grayscale images using a custom plug in (http:// bui2.win.ad.jhu.edu/telometer/) written for the program Image J (http://rsbweb.nih.gov/ij/). Quantitative telomere FISH was used to determine telomere length in tumor cells and associated stromal cells. The telomere fluorescence intensity was calculated as (total Cy3 intensity)/(total 4',6-diamidino-2-phenylindole intensity) for each sampled nucleus; thus, compensating for ploidy differences or differing nuclear fraction due to microtomy. Approximately 30 representative nuclei were analyzed for tumor 
cells and tumor stromal cells as described. ${ }^{23}$ The relative mean telomere length for tumor and stromal cells was calculated based on fluorescence signal from each case. The telomere index was then defined as the ratio of relative mean telomere length of tumor cells/stromal cells from each specimen.

\section{Statistical Analysis}

Telomere characteristics of the primary tumors, including mean cancer cell ratio, mean stromal cell ratio and telomere index among the four histologic types were compared using ANOVA. Comparisons between individual histologic types were performed using the Wilcoxon rank-sum test when the telomere index was treated as a continuous variable and the Fisher's exact test when the telomere index was treated as a categorical variable. The telomere index among matched pairs of primary and recurrent tumor tissue from the same patient was also compared using the Wilcoxon sign-rank test. Kaplan-Meier survival curves of both telomere index categories were plotted for each tumor type. A stratified log-rank test was used to test equality of the survivor functions. Hazard ratios for risk of dying were estimated using Cox regression models stratified by histological type and adjusted for age and stage at diagnosis among those in whom followup was available. For this analysis, the telomere index was treated as a binary variable, grouped as either $\leq 1$ or $>1$. Greater than 1 reflecting a larger average telomere length in the carcinoma cells than the stromal cells. Proportionality of the models was also assessed. $P=0.05$ was considered statistically significant. Stata 9 (College Station, TX, USA) was used to carry out the analyses. Correlation of the telomere index and specific histological features (for example cystic vs adenofibromatous clear cell carcinomas) was performed using KruskalWallis test.

\section{Results}

The mean patient age was 57 years (range $23-88$ years). Most of the ovarian carcinoma specimens were from primary tumors but in seven cases tissue from both primary and recurrent tumors was available for comparison (Table 1). All patients with high-grade serous carcinomas were stage III or IV. The median follow-up time for 196 cases with available follow-up information was 34 months (range: 0.1 to 166.8 ).

The telomere index in all samples analyzed is shown in Figure 1 and representative images of telomere FISH in different types of ovarian carcinoma are shown in Figure 2. Comparing the four different ovarian carcinoma histologic types (lowgrade serous, high-grade serous, low-grade endometrioid and clear cell carcinoma), the mean telomere index in clear cell carcinoma was significantly
Table 1 Clinico-pathological characteristics of ovarian cancer patients

\begin{tabular}{lc}
\hline Clinico-pathologic characteristics & $\mathrm{n}(\%)$ \\
\hline Ethnicity & \\
Caucasian & $95(44.8)$ \\
Asian & $81(38.2)$ \\
African-American & $11(5.2)$ \\
Missing & $25(11.8)$ \\
& \\
Cases origin & \\
JHMI & $128(60.4)$ \\
Treated at JHMI & $112(87.5)$ \\
Consults & $16(12.5)$ \\
NTUH & $84(39.6)$ \\
& \\
Tumor & $174(82.1)$ \\
Primary & $20(9.4)$ \\
Recurrence & $7(3.3)$ \\
Primary and recurrence & $11(5.2)$ \\
Not known & \\
Histotype & $99(46.7)$ \\
HGSC & $56(26.4)$ \\
CCC & $31(14.6)$ \\
EMC & $26(12.3)$ \\
LGSC & \\
& \\
Stage of primary & $61(28.8)$ \\
Stage I & $11(5.2)$ \\
Stage II & $98(46.2)$ \\
Stage III & $22(10.4)$ \\
Stage IV & $20(9.4)$ \\
Missing & \\
&
\end{tabular}

Abbreviations: CCC, clear cell carcinoma; EMC endometrioid carcinoma; HGSC, high-grade serous carcinoma; JHMI, Johns Hopkins Medical Institutions; LGSC, low grade serous carcinoma; n, number of cases; NTUH, National Taiwan University Hospital.

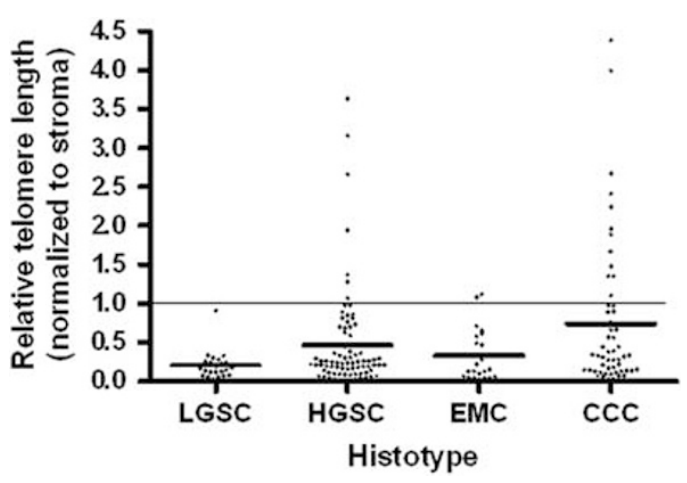

Figure 1 Telomere index in different histological types of ovarian carcinoma. The telomere index is defined as the ratio of telomere fluorescence intensity of tumor cells to corresponding stromal cells. Each symbol represents an individual specimen and the average of the index is shown as a bar for each type. When the telomere index was categorized into two groups, $(\leq 1$ vs $>1)$, clear cell carcinoma had more cases with a longer telomere (index $>1$ ) than the other types ( $P=0.01$, Fisher's exact test). LGSC: lowgrade serous carcinoma, HGSC: high-grade serous carcinoma, EMC: endometrioid carcinoma, CCC: clear cell carcinoma.

higher than that in other histologic types in combination (Figure 1; $P=0.007$ ). Similarly, if the telomere index $(\leq 1 v s>1)$ was used as a cutoff for the binary analysis, clear cell carcinoma had more 
cases with longer telomeres (index $>1$ ) than the other histologic subtypes (12 of 56 vs 9 of 164, respectively; $P=0.01$, Fisher's exact test). In contrast, neither low-grade serous nor high-grade serous and low-grade endometrioid carcinoma demonstrated a significant difference compared with the other ovarian carcinoma types $(P>0.05)$. We also analyzed the prognostic significance of telomere index in all the histologic types. After adjusting for age and clinical stage, the overall hazard ratio for death was 4.93 (95\% CI: 1.64 to $14.86, P=0.005$ ) for clear cell carcinomas with a telomere index $>1$ as compared with those with telomere index $\leq 1$ (Figure 3). The other cancer subtypes did not show similar findings $(P>0.05)$. We have reported that subdividing ovarian clear cell carcinomas into cystic and adenofibromatous reveals differences in a number of clinicopathological features including their association with endometriosis, histologic patterns, stage distribution and clinical behavior. ${ }^{25}$ We therefore correlated the telomere index in cystic $v s$ adenofibromatous clear cell carcinomas in 50 cases but there was no statistically significant difference between them $(P=0.955)$. Among 99 high-grade serous carcinomas, there were seven cases in which tissue from the primary and first recurrence in the same patient was available for analysis. Recurrent high-grade serous carcinomas tended to have shorter telomeres than the primary tumors, although the difference was not statistically significant $(P=0.09)$. All seven primary tumors except one (patient 4) had shorter telomeres in the recurrent tumors (Table 2).

\section{Discussion}

In view of the importance of telomere function in carcinogenesis, we hypothesized that measurement a
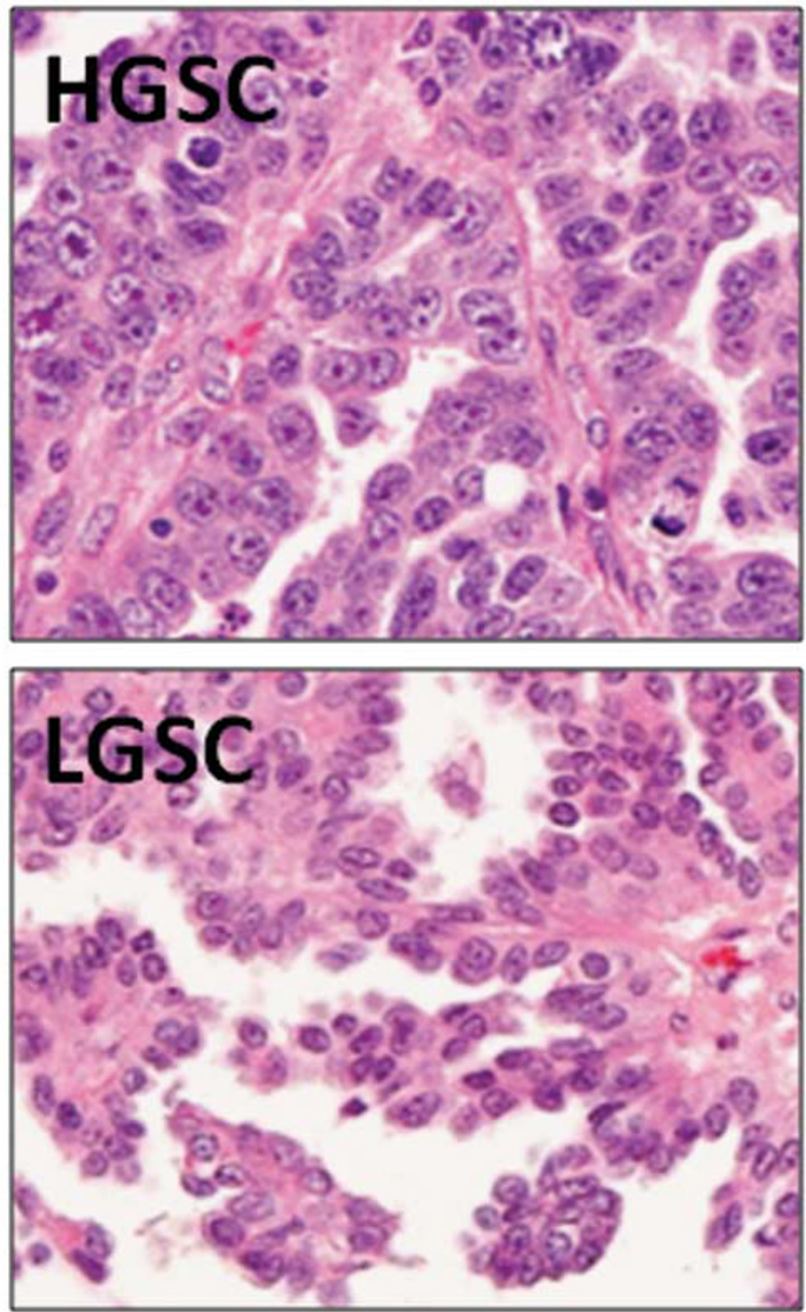
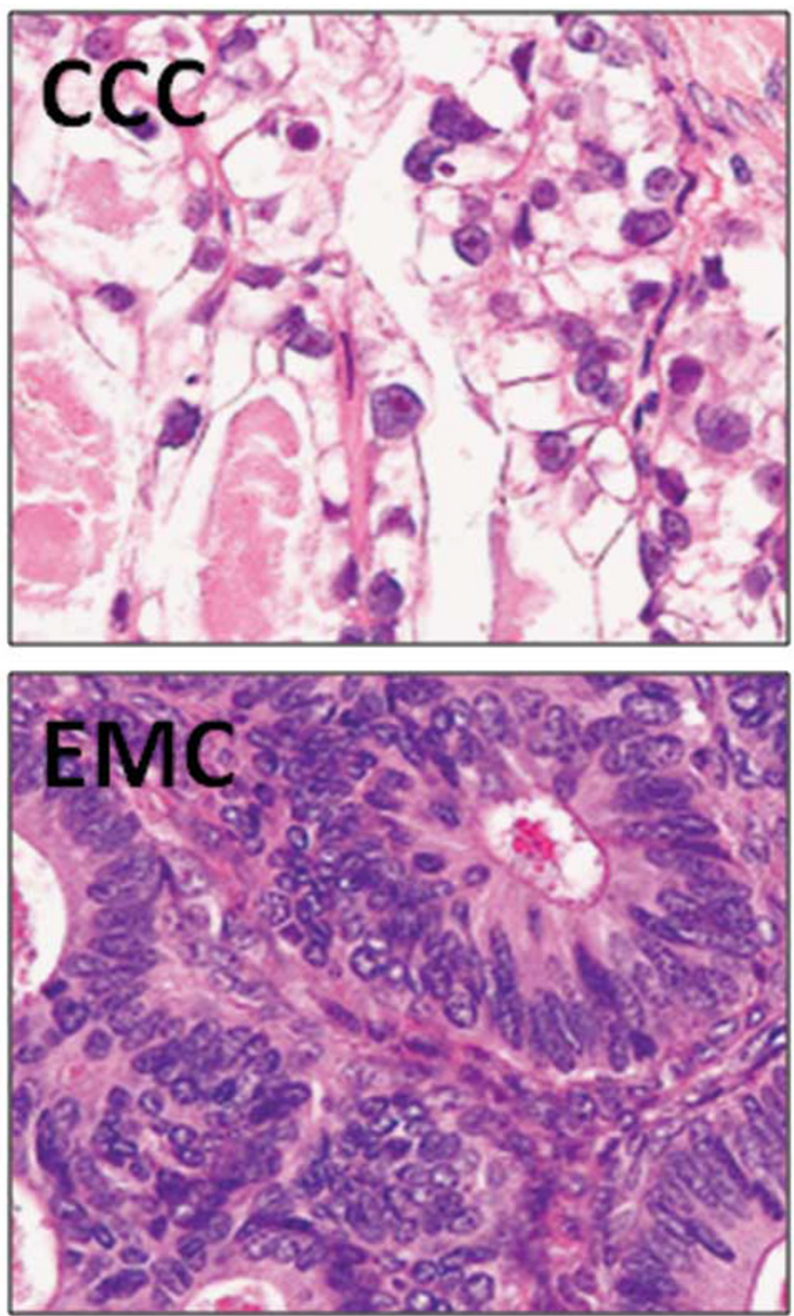

Figure 2 Representative images of telomere fluorescence in situ hybridization in different histological types of ovarian cancer. (a) Hematoxylin and eosin stained sections. (b) Telomere length is reflected by the total red fluorescence intensity in nuclei, which were counterstained with DAPI (blue florescence). In low-grade serous carcinoma (LGSC), high-grade serous carcinoma (HGSC) and endometrioid carcinoma (EMC) the stromal cells (filled arrows) show stronger red signal than carcinoma cells (open arrows) reflecting a smaller telomere index. In clear cell carcinoma (CCC), the carcinoma cells (open arrows) present longer telomere length as indicated by bigger, more numerous and intense red signal. 

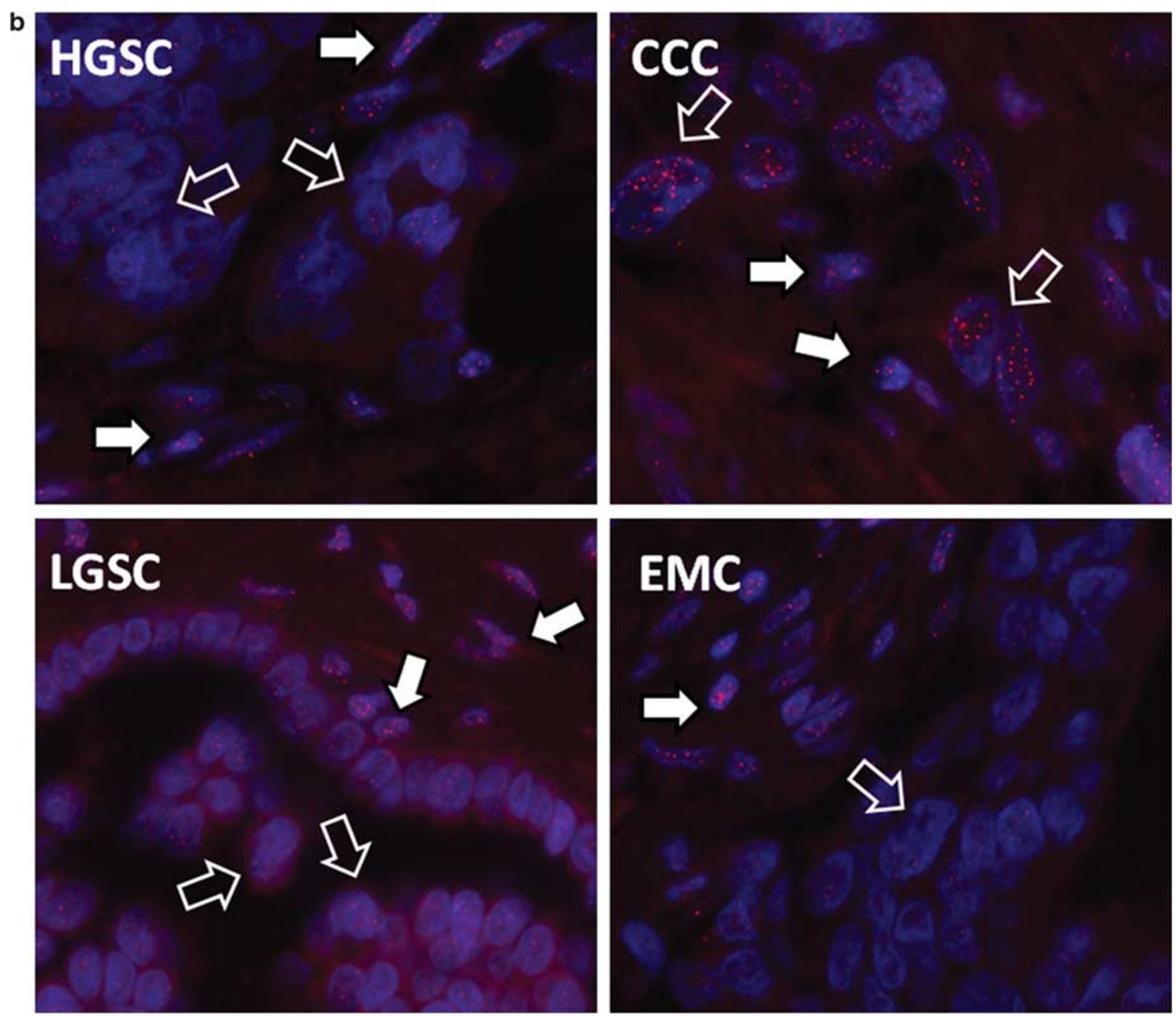

Figure 2 Continued.

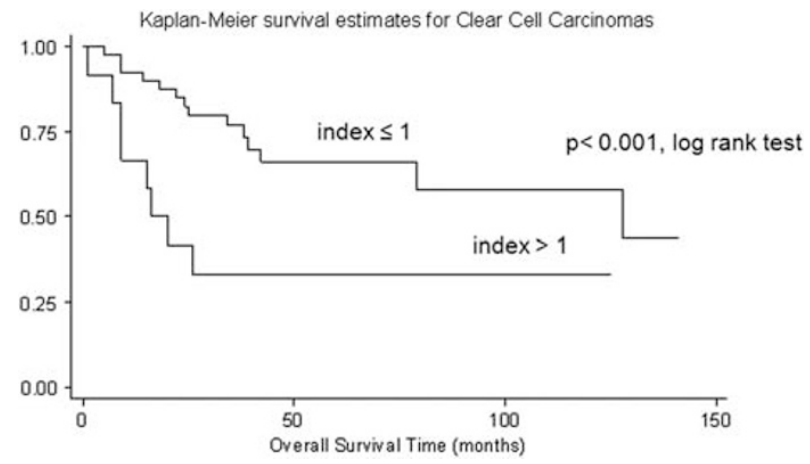

Table 2 Telomere index of matched primary and recurrent highgrade serous carcinomas

\begin{tabular}{lcc}
\hline Patient no & Primary & Recurrent \\
\hline 1 & 0.25 & 0.19 \\
2 & 1.07 & 0.47 \\
3 & 0.63 & 0.23 \\
4 & 0.00 & 0.29 \\
5 & 0.89 & 0.22 \\
6 & 0.21 & 0.14 \\
7 & 0.30 & 0.05 \\
\hline
\end{tabular}

Figure 3 Kaplan-Meier survival curve analysis of telomere index in clear cell carcinomas. After adjusting for the patients' age and clinical stage, patients with long telomeres (index $>1$ ) in their tumors had a worse overall survival than patients whose tumors had shorter telomeres (index $\leq 1)(P<0.001$, log rank test). The hazard ratio for death was 4.93 (95\% CI 1.64-14.86 $P=0.005)$ for women with clear cell carcinomas with a higher telomere index $(>1)$ compared with those women whose tumors had a smaller telomere index $(\leq 1)$.

of telomere length might provide clues to the pathogenesis of different types of ovarian carcinoma. In this study, we focused on telomere length, not

telomerase expression level and activity because telomere length, in contrast to telomere expression, in mammalian cells is regulated by a complex and dynamic process involving both telomerase-dependent and independent mechanisms. ${ }^{26}$ We found that ovarian clear cell carcinomas contained on average significantly longer telomeres than highgrade serous, low-grade serous and low-grade endometrioid carcinomas, a finding that confirms the distinct molecular genetic differences of the 
different ovarian carcinoma types and underscores the unique nature of ovarian clear cell carcinoma.

Although ovarian clear cell carcinoma represents $<10 \%$ of ovarian carcinoma in the United States, it occurs more frequently in Asian women and, in fact, its incidence appears to be increasing in this population. ${ }^{27,28}$ As compared to high-grade (conventional) serous carcinoma, women with clear cell carcinoma present at a younger age and at an earlier clinical stage. ${ }^{27,29,30}$ Also compared with high-grade serous carcinoma, advanced stage clear cell carcinoma is not as sensitive to conventional cytotoxic chemotherapeutic agents. ${ }^{31}$ The molecular genetic alterations that underlie the development of ovarian clear cell carcinoma are now beginning to emerge. The most common molecular genetic changes that have been identified thus far are a somatic inactivating mutation of $A R I D 1 A,,^{32,33}$ an activating mutation of $P I K 3 C A^{34}$ and deletion of PTEN, a tumor suppressor gene involved in the PIK3CA signaling pathway, ${ }^{35,36}$ which support the role of an aberrant PIK3CA pathway in the development of clear cell carcinoma. In addition, single nucleotide polymorphism array analysis has identified frequent amplification of the $Z N F 217$ (zinc finger protein 217) locus and deletion of the $C D K N 2 A / 2 B$ locus in clear cell carcinoma, suggesting that pathways involving these two genes are also important in the development of this tumor. ${ }^{37}$ Although both clear cell and endometrioid carcinomas are derived from endometriosis ${ }^{35}$ and share some molecular genetic features such as inactivating mutations of $A R I D 1 A$ and activating mutations of PIK3CA, they adopt different molecular programs for their development, as is evident by their distinctly different morphologic phenotype. For example, activating mutations in $\beta$-catenin and microsatellite instability, which occur frequently in endometrioid carcinoma, have only rarely been detected in clear cell carcinoma. ${ }^{2}$ The results from the current study provide additional evidence that telomere length in ovarian clear cell carcinoma is distinctly different from the other histologic types of ovarian carcinoma.

It is also worth noting that clear cell carcinoma in contrast to low-grade serous and low-grade endometrioid carcinomas, has a high level of inter-tumoral heterogeneity of telomere length as evidenced by the wide standard error (Figure 1), suggesting that there are subsets of clear cell carcinoma based on different telomere lengths. Telomerase inhibition enhances the response to anticancer $\operatorname{drugs}^{38}$ and telomere length may prove to be a useful prognostic feature in combination with other immunohistochemical markers. For example, IGF2BP3 (IMP3) expression has been reported to be an independent marker of reduced disease-specific survival in clear cell carcinoma, but not in high-grade serous or endometrioid carcinomas of the ovary. ${ }^{39}$ Similarly, enhanced expression of Annexin A4 in clear cell carcinoma and its association with chemoresistance to carboplatin has been recently reported. ${ }^{40}$
The lack of correlation of telomere length and clinical outcome in other types of ovarian carcinoma is intriguing. Although the finding suggests that the role of telomere length in clear cell carcinoma is different from other histologic types, more cases, particularly of low-grade serous and low-grade endometrioid carcinomas must be studied in a prospective manner to validate this conclusion. Another unrelated but interesting observation was that recurrent high-grade serous carcinomas tend to have shorter telomeres than the primary tumor but the number of cases in our study was small and therefore additional cases must be studied to confirm these findings.

In summary, this report is the first comprehensive investigation of telomere length among the major types of ovarian carcinoma. Using quantitative telomere FISH, we are able to precisely measure the mean relative telomere length and correlate it with various histologic types of ovarian carcinoma and with clinical outcome. Our findings showed that clear cell carcinomas have longer telomeres compared with the other histologic types of ovarian carcinoma. Additionally, longer telomeres in clear cell carcinoma were associated with poor outcome suggesting that aberrations in telomere length, in particular lengthening, may have an important role in the development and progression of this neoplasm.

\section{Disclosure/conflict of interest}

The authors declare no conflict of interest.

\section{References}

1 Gilks CB. Molecular abnormalities in ovarian cancer subtypes other than high-grade serous carcinoma. J Oncol 2010;740968.

2 Cho KR, Shih IM. Ovarian cancer. Annu Rev Pathol Mech Dis 2009;4:287-313.

3 Kobel M, Kalloger SE, Boyd N, et al. Ovarian carcinoma subtypes are different diseases: implications for biomarker studies. PLoS Med 2008;5:e232.

4 Shih I-M, Kurman RJ. Ovarian tumorigenesis- a proposed model based on morphological and molecular genetic analysis. Am J Pathol 2004;164:1511-1518.

5 Shih Ie M, Kurman RJ. Molecular pathogenesis of ovarian borderline tumors: new insights and old challenges. Clin Cancer Res 2005;11:7273-7279.

6 Kurman RJ, Shih IM. The origin and pathogenesis of epithelial ovarian cancer: a proposed unifying theory. Am J Surg Pathol 2010;34:433-443.

7 Ahmed AA, Etemadmoghadam D, Temple J, et al. Driver mutations in TP53 are ubiquitous in high grade serous carcinoma of the ovary. J Pathol 2010; 221:49-56.

8 Salani R, Kurman RJ, Giuntoli II R, et al. Assessment of TP53 mutation using purified tissue samples of ovarian serous carcinomas reveals a higher mutation rate than previously reported and does not correlate 
with drug resistance. Int J Gynecol Cancer 2008;18: 487-491.

9 Kuo KT, Guan B, Feng Y, et al. Analysis of DNA copy number alterations in ovarian serous tumors identifies new molecular genetic changes in low-grade and highgrade carcinomas. Cancer Res 2009;69:4036-4042.

10 Grobelny JV, Kulp-McEliece M, Broccoli D. Effects of reconstitution of telomerase activity on telomere maintenance by the alternative lengthening of telomeres (ALT) pathway. Hum Mol Genet 2001;10:1953-1961.

11 Batista LF, Artandi SE. Telomere uncapping, chromosomes, and carcinomas. Cancer Cell 2009;15:455-457.

12 Meeker AK, Hicks JL, Iacobuzio-Donahue CA, et al. Telomere length abnormalities occur early in the initiation of epithelial carcinogenesis. Clin Cancer Res 2004;10:3317-3326.

13 Kuhn E, Meeker A, Wang TL, et al. Shortened telomeres in serous tubal intraepithelial carcinoma: an early event in ovarian high-grade serous carcinogenesis. Am J Surg Pathol 2010;34:829-836.

14 Meeker AK, De Marzo AM. Recent advances in telomere biology: implications for human cancer. Curr Opin Oncol 2004;16:32-38.

15 Counter CM, Hirte HW, Bacchetti S, Harley CB. Telomerase activity in human ovarian carcinoma. Proc Natl Acad Sci USA 1994;91:2900-2904.

16 Kyo S, Kanaya T, Takakura M, et al. Expression of human telomerase subunits in ovarian malignant, borderline and benign tumors. Int J Cancer 1999; 80(6):804-809.

17 Braunstein I, Cohen-Barak O, Shachaf C, et al. Human telomerase reverse transcriptase promoter regulation in normal and malignant human ovarian epithelial cells. Cancer Res 2001;61:5529-5536.

18 Sun PM, Wei LH, Luo MY, et al. The telomerase activity and expression of hTERT gene can serve as indicators in the anti-cancer treatment of human ovarian cancer. Eur J Obstet Gynecol Reprod Biol 2007;130:249-257.

19 Wang SJ, Sakamoto T, Yasuda Si S, et al. The relationship between telomere length and telomerase activity in gynecologic cancers. Gynecol Oncol 2002;84:81-84.

20 Aldea AI, Barnadas A, Tarrats A, et al. Analysis of telomeric length in epithelial carcinoma of the ovary. Med Clin (Barc) 1998;110:561-565.

21 Murakami J, Nagai N, Ohama K, et al. Telomerase activity in ovarian tumors. Cancer 1997;80:1085-1092.

22 Murakami J, Nagai N, Ohama K. Telomerase activity and telomere length as diagnostic tumor marker for ovarian tumors. Nippon Rinsho 1998;56:1310-1315.

23 Meeker AK, Gage WR, Hicks JL, et al. Telomere length assessment in human archival tissues: combined telomere fluorescence in situ hybridization and immunostaining. Am J Pathol 2002;160:1259-1268.

24 Chen C, Hong YK, Ontiveros SD, et al. Single base discrimination of CENP-B repeats on mouse and human Chromosomes with PNA-FISH. Mamm Genome 1999;10:13-18.

25 Veras E, Mao TL, Ayhan A, et al. Cystic and adenofibromatous clear cell carcinomas of the ovary: distinctive tumors that differ in their pathogenesis and behavior: a clinicopathologic analysis of 122 cases. Am J Surg Pathol 2009;33:844-853.

$26 \mathrm{Kim} \mathrm{JH}$, Lee GE, Kim JC, et al. A novel telomere elongation in an adriamycin-resistant stomach cancer cell line with decreased telomerase activity. Mol Cells 2002;13:228-236.

27 Chan JK, Teoh D, Hu JM, et al. Do clear cell ovarian carcinomas have poorer prognosis compared to other epithelial cell types? A study of 1411 clear cell ovarian cancers. Gynecol Oncol 2008;109:370-376.

28 Ushijima K. Current status of gynecologic cancer in Japan. J Gynecol Oncol 2009;20:67-71.

29 Takano M, Kikuchi Y, Yaegashi N, et al. Clear cell carcinoma of the ovary: a retrospective multicentre experience of 254 patients with complete surgical staging. Br J Cancer 2006;94:1369-1374.

30 Mizuno M, Kikkawa F, Shibata K, et al. Long-term follow-up and prognostic factor analysis in clear cell adenocarcinoma of the ovary. J Surg Oncol 2006;94: 138-143.

31 Jenison EL, Montag AG, Griffiths CT, et al. Clear cell adenocarcinoma of the ovary: a clinical analysis and comparison with serous carcinoma. Gynecol Oncol 1989;32:65-71.

32 Jones S, Wang TL, Shih IM, et al. Frequent mutations of chromatin remodeling gene ARID1A in ovarian clear cell carcinoma. Science 2010;330:228-231.

33 Wiegand KC, Shah SP, Al-Agha OM, et al. ARID1A mutations in endometriosis-associated ovarian carcinomas. N Engl J Med 2010;363:1532-1543.

34 Kuo KT, Mao TL, Jones S, et al. Frequent activating mutations of PIK3CA in ovarian clear cell carcinoma. Am J Pathol 2009;174:1597-1601.

35 Sato N, Tsunoda $\mathrm{H}$, Nishida $\mathrm{M}$, et al. Loss of heterozygosity on 10q23.3 and mutation of the tumor suppressor gene PTEN in benign endometrial cyst of the ovary: possible sequence progression from benign endometrial cyst to endometrioid carcinoma and clear cell carcinoma of the ovary. Cancer Res 2000;60: 7052-7056.

36 Ho CM, Lin MC, Huang $\mathrm{SH}$, et al. PTEN promoter methylation and LOH of 10q22-23 locus in PTEN expression of ovarian clear cell adenocarcinomas. Gynecol Oncol 2009;112:307-313.

37 Kuo K, Mao T, Feng Y, et al. DNA copy number profiles in affinity-purified ovarian clear cell carcinoma. Clin Cancer Res 2010;16:1997-2008.

38 Cerone MA, Londono-Vallejo JA, Autexier C. Telomerase inhibition enhances the response to anticancer drug treatment in human breast cancer cells. Mol Cancer Ther 2006;5:1669-1675.

39 Kobel M, Xu H, Bourne PA, et al. IGF2BP3 (IMP3) expression is a marker of unfavorable prognosis in ovarian carcinoma of clear cell subtype. Mod Pathol 2009;22:469-475.

40 Aoki D, Oda Y, Hattori S, et al. Overexpression of class III beta-tubulin predicts good response to taxane-based chemotherapy in ovarian clear cell adenocarcinoma. Clin Cancer Res 2009;15:1473-1480. 